\title{
Low genetic diversity in an endangered species: recent or historic pattern?
}

\author{
Marjorie D. Matocq , Francis X. Villablanca
}

\begin{abstract}
Examining patterns of genetic diversity has become an integral component of many management plans concerning endangered species, yet interpreting the processes underlying such patterns remains challenging. We demonstrate low genetic diversity in a critically endangered small mammal population. A common interpretation of this pattern would be that it is the result of a known, recent decline in this population. We test this interpretation and find it to be incorrect. Instead, by using museum voucher specimens, we show that the pattern of low genetic diversity is historical. This study demonstrates the importance of choosing appropriate reference groups by which to interpret modern levels of genetic diversity in endangered species. We conclude that analysis of archival specimens may be essential in cases where genetic diversity is driving conservation management decisions because it may allow us to distinguish the effects of low genetic diversity from the process of losing diversity. We recognize that this approach can be limited due to several sampling issues: archival material may not be available, statistical power needs to be evaluated, sample sizes and sequence lengths may be suboptimal due to intrinsic difficulties associated with amplification of degraded DNA. These issues are discussed and possible solutions identified.
\end{abstract}

\section{Introduction}

Declines in genetic variation due to recent demographic bottlenecks in wild populations are of great concern to conservation biologists (Hedrick and Miller, 1992; Lacy, 1997). This concern is based in population genetic theory that predicts lower individual fitness and lower population adaptability as consequences of reduced genetic variation (Lande, 1988). Reduction of a population's genetic diversity below historic, equilibrium levels is expected to have detrimental effects because such reductions are accompanied by inbreeding (Lacy, 1997) (defined here as an increase in the average relatedness of breeding individuals, Wright, 1969). Empirical studies support the hypothesis that increased inbreeding lowers individual fitness and the probability of population persistence (O’Brien et al., 1987; Ralls et al., 1988; Ballou, 1997; Newman and Pilson, 1997; Saccheri et al., 1998).
Multiple factors are implicated in species extinctions, including habitat loss, habitat fragmentation, demographic instability, inbreeding and other genetic factors (Lande, 1988). If one of these factors, namely recent changes in genetic diversity and inbreeding, can be tested and perhaps rejected, then research and management can focus on other extinction mechanisms. Furthermore, differentiating patterns of low genetic diversity from the process of losing diversity should allow for testing the effects of each separately.

Genetic diversity surveys in endangered populations typically determine the variation currently maintained in the population rather than the magnitude or rate of loss of genetic diversity over time. Finding evidence of temporal changes in genetic diversity is challenging. Difficulties include finding appropriate molecular markers, methods of analysis (Luikart, et al. 1998), and establishing a baseline or reference point against which to measure change (Taylor et al., 1994; Steinberg and Jordan, 1998). Only recently (1998) has a reduction in genetic diversity for an endangered species been demonstrated empirically (Bouzat et al., 1998). 
In attempts to quantify changes in genetic diversity for endangered populations, it is essential to establish a baseline representing the pre-bottleneck conditions. Researchers often are forced to evaluate diversity in other populations of the same species or of closely related species and use these non-endangered samples as reference points (Hoelzel et al., 1993; Merola, 1994; Taylor et al., 1994; Houlden et al., 1996; Nusser et al., 1996; Bouzat et al., 1998; Steinberg and Jordan, 1998). In designating such samples as reference points it is assumed they represent the genetic diversity in the endangered group prior to the event(s) that led to a population reduction and endangered status. This assumption can only be valid if the endangered and reference populations have identical demographic histories and reflect no differences in genetic diversity other than those caused by a demographic crash. When the endangered and reference populations share a common demographic history then, under the neutral model of molecular evolution, they would have the same prebottleneck effective population size $\left(N_{\mathrm{e}}\right)$ and statistically indistinguishable levels of pre-bottleneck genetic diversity [i.e. as measured by $\theta=N_{\mathrm{e}} \mu$, and estimated by nucleotide diversity (Slatkin, 1987; Kuhner et al., 1998)]. A population is defined here as a geographic unit that is not genetically subdivided (i.e. non-significant $F_{\mathrm{ST}}$, Wright, 1969). Importantly, genetic diversity surveys reveal heterogeneity in levels of genetic variability (and demographic history) between populations of genetically subdivided species (Lewontin, 1991). This is evidence that population samples, even of a single species, may be invalid reference points of pre-bottleneck genetic diversity. Two samples may provide valid reference points: (1) an actual sample from the pre-bottleneck population or (2) a sample of any population descended from the pre-bottleneck population that has not experienced change in genetic diversity since that time. Due to the genetic heterogeneity possible within species it is difficult to justify using any interspecific reference points.

We argue that use of archival (e.g. museum or herbarium) specimens may allow for a powerful test of loss in genetic diversity over time (Thomas et al., 1990; Wayne and Jenks, 1991; Villablanca, 1994; Bouzat et al., 1998). If an archival reference sample represents genetic variation found in a population prior to the event(s) leading to endangered status, such a sample would provide the most appropriate reference against which to measure current levels of diversity. We explore this approach using an endangered subspecies of heteromyid rodent [Dipodomys heermanni morroensis, the Morro Bay kangaroo rat (Merriam, 1907)]. This subspecies occurs as a single population in a small sand dune complex (567 ha.) along the coast of Central California (Stewart and Roest, 1960). Historic genetic subdivisions are considered very unlikely due to the small and contiguous nature of the suitable habitat (Stewart and
Roest, 1960). This habitat is geographically isolated from that of other populations (subspecies) by approximately 10 $\mathrm{km}$. The population size in 1957 (and presumably earlier) was estimated to be 8000 individuals (Stewart and Roest, 1960). By 1986, however, only 50 individuals remained with available habitat having been reduced by $98 \%$ to 12.6 ha. (US Fish and Wildlife Service, 1995). This dramatic decrease in population size raised concerns regarding the genetic viability of the population and how a potential increase in inbreeding would affect its recovery (US Fish and Wildlife Service, 1995). Here, we test whether low genetic diversity is due to a recent population decline. We find that a lack of diversity can be historical and not necessarily due to recent demographic fluctuations.

\section{Methods}

We sequenced a $317 \mathrm{bp}$ fragment of the control region and a $126 \mathrm{bp}$ fragment of the cytochrome $\mathrm{b}$ gene of the mitochondrial genome isolated from post-bottleneck $D$. h. morroensis samples collected in 1986-1987 $(N=8)$. We sequenced the same regions in a sample $(N=8)$ of Dipodomys heermanni arenae, the Lompoc kangaroo rat, which is presumably the most closely related subspecies and is the geographically most proximate population of kangaroo rat to the Morro Bay population (Hall, 1981). Finally, because a series of dried study skins were made when the Morro Bay population was studied in 1918, we were able to analyse the same $443 \mathrm{bp}$ in a pre-bottleneck sample $(N=8)$. All methods have been modified to provide redundant tests for cross contamination in archival samples.

Small pieces of tissue $\left(2 \mathrm{~mm}^{2}\right)$ taken from dried study skins were digested overnight at $55^{\circ} \mathrm{C}$ in $500 \mu \mathrm{l}$ of 10 $\mathrm{mM}$ Tris- $\mathrm{HCl}, 35 \mathrm{mM}$ DTT, $50 \mu \mathrm{g} / \mu \mathrm{l}$ proteinase $\mathrm{K}$, and $0.9 \%$ Laureth 10 (Mazer Chemicals). We added 5\% Chelex (Biorad) to each sample, incubated at $55^{\circ} \mathrm{C}$ for $30 \mathrm{~min}$ then heated to $95^{\circ} \mathrm{C}$ for $10 \mathrm{~min}$. The Chelex beads were pelleted by centrifugation for $2 \mathrm{~min}$ and the supernatent stored at $-20^{\circ} \mathrm{C}$. Only three samples were extracted at a time to reduce risk of contamination and loss of tissue in the event of contamination. At least two negative controls (mock extractions) were carried out during each extraction experiment. All pre-amplification procedures were conducted in a room isolated from post-amplification manipulations. All equipment and reagents were dedicated to pre-amplification procedures and all work surfaces and implements were bleached immediately prior to use. Initial extractions were conducted in a laboratory where no previous work with kangaroo rat tissue had been performed. Extractions from historic tissues were completed prior to commencing work with modern tissues. Sequences for multiple individuals were replicated in two independent labs 
(MM at UCB and FV at University of Hawaii). The only reagents shared between labs were the initial DNA extractions.

Three separate primer pairs were used to amplify and sequence small portions of the entire control region fragment analysed here: TAS-Dpd3, Dpd4-Dpd7, Dpd6TDKD (Thomas et al., 1990; Villablanca, 1994), Dpd7 5'-TACCATCCTCCGTGAAACCA-3', Dpd6 5'-TCCTTGTCCATATGACTATC-3', TDKD 5'-CATCTGGTTCCTACTTCAGG-3' (TDKD designed by Tom Kocher). A single primer pair MVZ05 5'-CGAAGCTTGATATGAAAAACCATCGTTG-3' and CGL2 5'AATAGRCCTGARGCGATTTGRAT-3' amplified the $126 \mathrm{bp}$ cytochrome $\mathrm{b}$ fragment. All primer pairs resulted in amplicons shorter than 200 base pairs. Routine amplification of fragments in excess of 200 bp was not possible. This phenomenon is to be expected with the degraded state of DNA characteristic of historic samples (Lindahl, 1993). Primers named "Dpd" or "CGL" are kangaroo rat specific and constitute at least one primer in each pair. Human DNA was used as a control in each amplification experiment. Primer pairs either did not amplify human DNA or amplified fragments of a different size than those obtained in kangaroo rats. Negative controls with no added DNA were run in each amplification experiment, as were the negative controls from each extraction. DNA amplifications were carried out in 25 $\mu 1$ reactions including $1 \times$ PCR Buffer with $1.5 \mathrm{mM}$ $\mathrm{MgCl}_{2}$ (Boeringer Mannheim), $0.2 \mathrm{mM}$ of each dNTP, $1.0 \mu \mathrm{M}$ of each primer, $10 \%$ BSA, $1.0 \mathrm{U}$ of Taq DNA polymerase (Boeringer Mannheim). Amplifications were performed in a PTC-100 thermocycler (MJ Research, Inc.) with the following conditions: $93^{\circ} \mathrm{C}, 3 \mathrm{~min}$ then 38 cycles of $93^{\circ} \mathrm{C}, 1 \mathrm{~min} ; 55^{\circ} \mathrm{C}, 1 \mathrm{~min}$ and $72^{\circ} \mathrm{C}, 1 \mathrm{~min}$. To avoid formation of primer-dimers, the primers and dNTP's were added to the reaction when samples had reached $85^{\circ} \mathrm{C}$. Double-strand DNA products were purified using the QIAquick PCR Purification kit (Qiagen), or GeneClean (Bio 101). Double-strand product was cycle-sequenced in $10 \mu \mathrm{l}$ final volume with TaqFS or dRhodamine and run on a 377 Automated Sequencer according to manufacturer specifications (Applied Biosystems, Inc.). Sequences were edited in the program Sequence Navigator (Applied Biosystems, Inc.) and Sequencher (GeneCodes).

Maximum likelihood (ML) estimates of nucleotide diversity $(\theta)$ were obtained from the computer package FLUCTUATE (Kuhner et al., 1998). The search was conducted with 10 short chains of 16 or 20 step increments and 16,000 or 20,000 steps, and 2 long chains of 16 or 20 step increments and 160,000 or 200,000 steps. The searches that resulted in estimates of nucleotide diversity near zero varied considerably in their estimates of $\theta$, unless one short chain (generally 8000 steps) was generated first and then used in a longer chain (generally 80,000 steps). In all such cases, the estimate of nucleotide diversity was on the same order of magnitude as Watterson's estimate (Watterson, 1975).

For mitochondrial data, $\theta_{\mathrm{mt}}$ is defined as the product of the female effective population size $\left(N_{\mathrm{F}}\right)$ and the mutation rate $(\mu)$. Estimates of population parameters based on $\theta_{\mathrm{mt}}$ assume haploid inheritance and neutrality. Selection on mitochondrial haplotypes within samples was tested using Tajima's D (Tajima, 1989). This test is valid for determining whether haplotypes within a population show evidence of selection, yet it does not have the power to identify selective sweeps.

\section{Results}

In the modern, post-bottleneck sample from Morro Bay $(N=8)$ seven of eight individuals shared the same mtDNA control region haplotype, while one individual had a haplotype that differed by a single transition. We found no variability in the cytochrome $b$ sequences (443 bp combined,Table 1). We compared these data to the genetic variation found in what is considered to be the most closely related subspecies and geographically proximate population of kangaroo rat, (Dipodomys heermanni arenae) (Hall, 1981). In the sample of $D . h$. arenae $(N=8)$ we found 6 distinct haplotypes with 15 polymorphic sites in the control region and 5 haplotypes with 4 polymorphic sites in cytochrome $b$ (Table 1). No haplotypes were shared between these two subspecies. Given the lack of polymorphism in the Morro Bay population at the cytochrome $\mathrm{b}$ gene, we restrict the following analysis to the control region data.

Assuming neutrality, any differences in genetic diversity observed across populations of kangaroo rats should reflect differences in demographic histories, given no evidence for selection of mitochondrial haplotypes in this sample (Tajima's D $P>0.05$ in all samples). Maximum likelihood estimates of $\theta_{\mathrm{mt}}$ (hereafter referred to as $\theta)$ showed a significant difference $(P<0.05)$ in genetic diversity of control region sequences between the endangered D. h. morroensis and D. h. arenae $[\theta=0.0013$ $($ S.E. $=0.0003)$ and $\theta=0.0774 \quad(0.0115)$ respectively]. Using only $D . h$ arenae as a reference, we would have concluded that the paucity of genetic diversity in the endangered population most likely resulted from the recent population crash (Fig. 1A).

Sequence data from the 1918, pre-bottleneck sample revealed only two haplotypes and one variable site, again, with no variation in cytochrome $b$ (Table 1). One haplotype was unique due to a single transition; all others matched the common haplotype from the postbottleneck sample. Estimates of nucleotide diversity are indistinguishable between pre- and post- bottleneck populations; the means and standard errors are identical between the samples $[\theta=0.0013$ (S.E. $=0.0003$ ) since only one haplotype varied at alternate sites in each 
Table 1

MtDNA haplotypes and polymorphic sites found in an endangered (D.h. morroensis) and non-endangered (D.h. arenae) kangaroo rat population ${ }^{\mathrm{a}}$

\begin{tabular}{|c|c|c|c|c|c|c|c|c|c|c|c|c|c|c|c|c|c|}
\hline \multirow{2}{*}{$\begin{array}{l}\text { Population } \\
\text { (A) }\end{array}$} & \multirow[t]{2}{*}{ Haplotype } & \multirow[t]{2}{*}{$N$} & \multicolumn{15}{|c|}{ Nucleotide position } \\
\hline & & & & & & & & & & & & & & & & & \\
\hline \multirow{3}{*}{$\begin{array}{l}\text { D. h. morroensis } \\
\text { Modern }\end{array}$} & & & 4 & 17 & 46 & 62 & 79 & 81 & 84 & 86 & 95 & 102 & 115 & 116 & 122 & 156 & 198 \\
\hline & Dlp1 & 7 & $\mathrm{C}$ & $\mathrm{T}$ & $\mathrm{C}$ & $A^{*}$ & $\mathrm{~T}$ & $\mathrm{C}$ & $\mathrm{C}$ & $\mathrm{C}$ & A & $\mathrm{T}$ & $\mathrm{A}$ & $\mathrm{A}$ & $\mathrm{C}$ & A & $\mathrm{C}$ \\
\hline & Dlp2 & 1 & . & $\mathrm{C}$ & . & . & . & . & . & . & . & . & . & . & . & . & . \\
\hline \multirow[t]{2}{*}{ Museum } & Dlp1 & 7 & . & . & . & . & . & . & . & . & . & . & . & . & . & . & . \\
\hline & Dlp3 & 1 & . & . & . & . & . & . & . & . & . & $\mathrm{C}$ & . & . & . & . & . \\
\hline \multirow[t]{6}{*}{ D. h. arenae } & Dlp4 & 2 & $\mathrm{C}$ & $\mathrm{C}$ & $\mathrm{C}$ & A & $\mathrm{T}$ & $\mathrm{T}$ & $\mathrm{C}$ & $\mathrm{T}$ & A & $\mathrm{T}$ & G & A & $\mathrm{C}$ & A & $\mathrm{C}$ \\
\hline & Dlp5 & 1 & . & . & $\mathrm{T}$ & G & $\mathrm{C}$ & $\mathrm{C}$ & . & $\mathrm{C}$ & G & $\mathrm{C}$ & A & . & . & G & . \\
\hline & Dlp6 & 2 & . & $\mathrm{T}$ & . & . & . & . & . & $\mathrm{C}$ & . & $\mathrm{C}$ & A & . & . & . & . \\
\hline & Dlp7 & 1 & . & . & . & . & . & . & . & $\mathrm{C}$ & . & . & . & . & . & . & . \\
\hline & Dlp8 & 1 & $\mathrm{~T}$ & . & . & . & . & $\mathrm{C}$ & . & $\mathrm{C}$ & . & $\mathrm{C}$ & A & . & $\mathrm{T}$ & . & $\mathrm{T}$ \\
\hline & Dlp9 & 1 & . & . & . & . & . & . & $\mathrm{T}$ & $\mathrm{C}$ & . & . & A & G & $\mathrm{T}$ & . & . \\
\hline \multicolumn{18}{|l|}{ (B) } \\
\hline D. h. morroensis & & & 24 & 48 & 57 & 60 & 69 & & & & & & & & & & \\
\hline Modern & Cyt.b1 & 8 & $\mathrm{C}$ & $\mathrm{T}$ & $\mathrm{T}$ & $\mathrm{T}$ & $\mathrm{C}$ & & & & & & & & & & \\
\hline Museum & Cyt.b1 & 8 & . & . & . & . & . & & & & & & & & & & \\
\hline \multirow[t]{5}{*}{ D. h. arenae } & Cyt.b2 & 3 & $\mathrm{~T}$ & $\mathrm{C}$ & $\mathrm{T}$ & $\mathrm{T}$ & $\mathrm{C}$ & & & & & & & & & & \\
\hline & Cyt.b3 & 2 & $\mathrm{C}$ & . & . & . & . & & & & & & & & & & \\
\hline & Cyt.b4 & 1 & . & . & . & $\mathrm{C}$ & . & & & & & & & & & & \\
\hline & Cyt.b5 & 1 & $\mathrm{C}$ & . & $\mathrm{C}$ & . & . & & & & & & & & & & \\
\hline & Cyt.b6 & 1 & $\mathrm{C}$ & . & . & . & $\mathrm{T}$ & & & & & & & & & & \\
\hline
\end{tabular}

a Dots indicate identity with the first sequence shown for each subspecies. *(A) Control region sequences with position 1 corresponding to position 15307 of the mouse mitochondrial genome. (B) Cytochrome b sequences with position 1 corresponding to position 14139 of the mouse mitochondrial genome.

data set (Fig. 1A and B). The pre-bottleneck (1918) sample shows that the near-zero level of genetic diversity in mtDNA is historical and not associated with the most recent population crash.

The interpretation of no change in genetic diversity over time is based on not rejecting the null hypothesis. As such, it is appropriate to ask whether our analysis has the statistical power to resolve differences if they do exist. This problem was explored through a computer simulation. We tested whether it would have been possible to detect a loss of genetic diversity if the historical diversity had been 5 times or even just 2 times greater. Simulated data sets were obtained by generating one random sequence with the length and nucleotide frequency composition observed in D. h. morroensis (SeqGen Ver. 1.1, Grassly et al., 1997) and duplicating it 8 times (sample size of $D . h . m$.). One randomly selected sequence (from the 8 ) was assigned either 2 or 5 polymorphic sites to simulate 2 and 5 times the observed polymorphism in our D. h. morroensis sample. Each set of 8 sequences was constrained so that only one individual varied at each of the segregating sites. Making more than one individual polymorphic at each segregating site would have resulted in a higher $\theta$ and even greater statistical power. Ten replicate data sets were generated for each scenario (2-fold and 5-fold). The maximum likelihood estimate of $\theta$ was obtained for each replicate (using FLUCTUATE, Kuhner et al.,
1998) and the value selected was the one with the lowest log likelihood (best estimate). Replicates were not significantly different from each other based on comparisons of the likelihood surfaces within 2 standard errors. The hypotheses tested were whether the observed nucleotide diversity in the endangered population was significantly lower than a simulated 5-fold or 2-fold excess in nucleotide diversity. The $95 \%$ confidence interval (standard error of the mean) of neither simulation includes the mean of the endangered sample (Fig. 2). Given the observed nucleotide diversity in our modern sample of $D$. $h$. morroensis, then, we could have rejected the null hypothesis of no change in genetic variation given a $50 \%$ or greater reduction in genetic diversity between the temporal samples of $D$. h. morroensis. The effect of the mutational model was explored by estimating nucleotide diversity for the D. h. morroensis data under two substitution models. These models differed in their transition bias (TS:TV ratio of 10:1 and 2:1). The mean nucleotide diversity values are identical, only the range of the confidence intervals differs (Fig. 2).

With the observed pre-bottleneck nucleotide diversity and its variance, our analysis would be able to recognize as significant a $50 \%$ reduction in genetic diversity between samples (in 95\% of cases). This statistical power is considered high, given the small number of alleles observed at a single locus (Luikart et al., 1998), and is not an artefact of the mutational model used in 


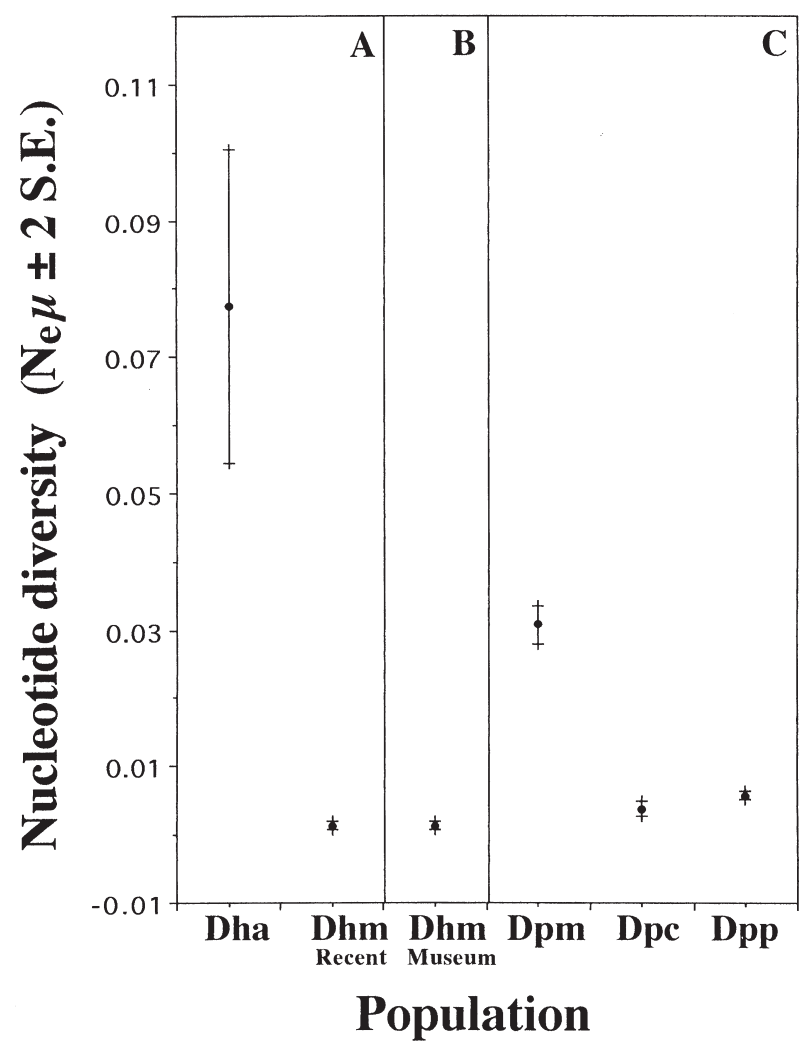

Fig. 1. Maximum likelihood estimates of nucleotide diversity (plus and minus two standard errors) from partial mitochondrial control region sequences. Mean values (dots) are for $\theta\left(\theta=N_{\mathrm{e}} \mu\right.$ as estimated by nucleotide diversity). The tic marks on the vertical bars indicate $95 \%$ confidence intervals of the population means. Panel A compares a recent sample of the endangered subspecies (Dipodomys heermanni morroensis - Dhm, $N=8$ ) to it's presumed closest geographic and taxonomic relative (Dipodomys heermanni arenae - Dha, $N=8$ ). This panel represents the type of comparison that is generally presented for endangered species. Panel B shows the estimated nucleotide diversity for a sample of Dhm $(N=8)$ obtained from a museum collection which predates the recent demographic bottleneck. This result, when compared to panel $\mathrm{A}$, indicates that, at least for this locus, low genetic diversity is historical. Panel $\mathrm{C}$, when compared to A, demonstrates that there is significant variation in nucleotide diversity between populations of kangaroo rats across species [i.e. Dipodomys panamintinus mohavensis-Dpm, D. p. caudatus- Dpc, and D. p. panamintinus- Dpp (Villablanca, 1994)] providing multiple opportunities for spurious comparisons. The large standard error values for Dha are a result of a lower sample size $(N=8)$ and larger absolute value of $\theta$ than those available for the panamintinus samples $(N=20)$.

estimating $\theta$ (Fig. 2). Also, it is unlikely that our samples are biased towards groups of closely related individuals. The same pattern of low haplotype diversity is found in both samples and is due to a single common haplotype. Finally, it is possible that not enough time has elapsed for the post-bottleneck population to lose diversity. Yet, even if the endangered population has not lost all of the diversity that it eventually will, clearly, the absolute level of nucleotide diversity was very low prior to the bottleneck.

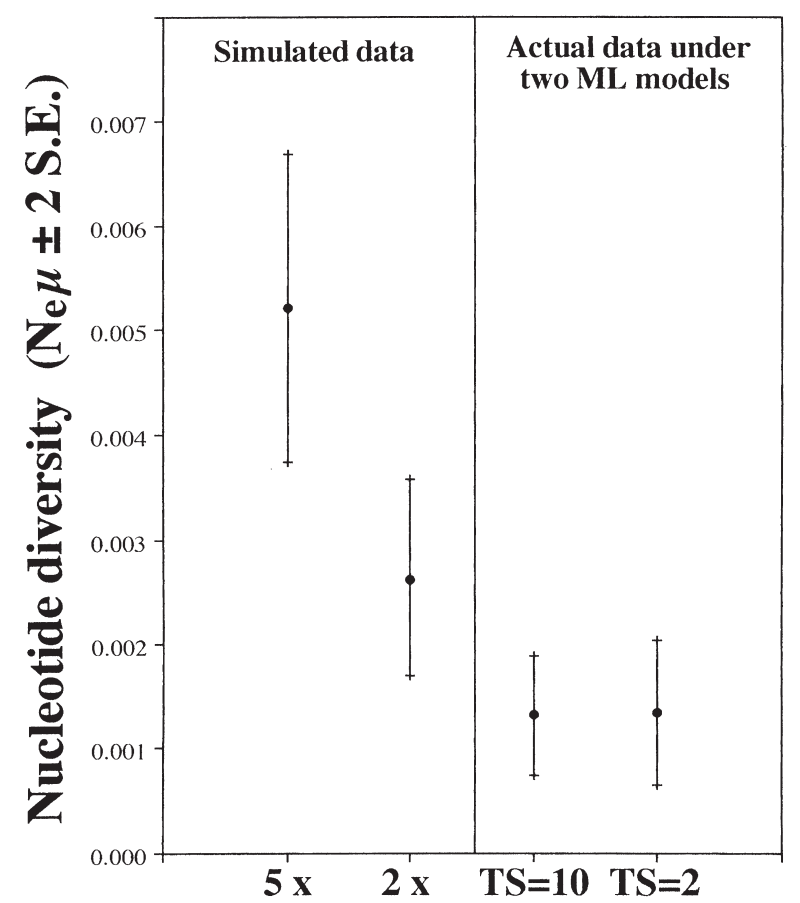

Fig. 2. Maximum likelihood estimate of nucleotide diversity (plus and minus two standard errors of the mean) from: (a) simulated data sets that contain 5 times and 2 times the number of polymorphic sites observed in our D.h.morroensis sample and, (b) actual data from partial mitochondrial control region sequences of $D$. h. morroensis under two maximum likelihood models of evolution (transitions (TS) weighted 10:1 and 2:1 relative to transversions). Tic marks indicate $95 \%$ confidence intervals of the population means. Given the observed nucleotide diversity in the $D$. h. morroensis samples (ML model $\mathrm{TS}=10$ and Fig. 1A), we could have rejected the null hypothesis of no change in genetic variation given a $50 \%$ or greater reduction in genetic diversity between the temporal samples of $D$. h. morroensis analyzed. This would be equivalent to the historical sample being twice as variable (Simulation $2 \times$ ) as the contemporary (endangered) sample. Variation in the mutational model employed has no effect on the results presented here.

To further explore the effect that reference populations have on inferences regarding loss of genetic variation over time, using the same fragment of mtDNA (Villablanca, 1994) we compared genetic diversity in $D$. h. morroensis to that in 3 populations of other kangaroo rat species (Fig. 1C). The broad range of values we obtained illustrates the difficulty of defining a "characteristic" level of population genetic diversity and underscores the need for appropriate temporal reference samples.

\section{Discussion}

Using the museum sample as our reference, we interpret low genetic diversity in the Morro Bay kangaroo rat to be historic and not associated with the most recent population decline. This conclusion is qualita- 
tively different from one based on comparing the modern level of genetic diversity in the endangered population to that found in the presumably closely related population of $D$. h. arenae. Further, our study shows that the choice of reference groups [intra- or interspecific (Fig. 1)] can drastically alter the interpretation of natural levels of genetic diversity in endangered populations. Our study also shows that correlating a loss of genetic diversity with a recent population crash is difficult when the prebottleneck diversity is already near zero. With low historic diversity, demonstrating further losses may be impossible given any single marker currently available, and may require large numbers of markers (Luikart et al., 1998).

The molecular marker(s) used in surveys of genetic diversity influences interpretations of current and historic demographic patterns (Luikart et al., 1998). The utility of mitochondrial DNA arises from an effective population size that is generally $1 / 4$ that of nuclear DNA coupled with a synonymous substitution rate that is an order of magnitude greater than the nuclear rate (Birky et al., 1989; Li and Graur, 1991). The mitochondrial locus is expected to reflect changes in effective population size due to bottlenecks in $1 / 4$ the time required by nuclear loci and therefore seems most appropriate for evaluations of change in genetic diversity over short time scales. Mitochondrial DNA, as a single locus, is most likely to result in rejection of the null hypothesis of no genetic change between pre- and post-bottleneck populations. Although mtDNA has been used widely in surveys of genetic diversity, its use in conservation biology has been criticized (Moritz, 1994). The strongest argument against the use of mitochondrial data alone is that they represent a single locus. As such, our inference of historical inbreeding and low diversity can technically only be applied to the mtDNA locus. Importantly, our hypothesis is testable using independent nuclear loci. Data from nuclear loci should also allow us to further test the alternative hypothesis that the pattern of low diversity at this locus is the result of a selective sweep.

The addition of nuclear data may supplement our view of demographic history in this subspecies, yet, the low historical mtDNA diversity remains a significant pattern. Surprisingly, a pattern of low genetic diversity is not exceptional. Examples of many mammalian populations with comparably low levels of genetic diversity can be found in the literature. Estimates of nucleotide diversity derived from sequence or RFLP analyses of mtDNA in 106 mammalian populations range from 0.0000 to $0.0508 \quad$ (mean $=0.0111$, S.D. $=0.0140$ ) (Avise et al., 1983; Patton et al., 1994; Patton and Smith, 1994; Taberlet et al., 1994; Maldonado et al., 1995; McKnight, 1995; Arctander et al., 1999; Petit et al., 1999; Wilmer et al., 1999). The distribution of these 106 values is not uniform since $38 \%$ of the values are zero. Although it is possible that some of these cases of low genetic diversity are due to recent demographic crashes, it is unlikely they can all be explained by recent demographic events. We suggest that patterns of low genetic diversity could be interpreted as reflecting a recent reduction in diversity, or alternatively, a historical lack of diversity. We acknowledge that a demographic crash can result in a loss of genetic diversity, but we argue that some endangered populations may have a history of small effective population sizes (or historical population crashes). Low historical genetic diversity is a viable, but rarely considered alternative hypothesis. Consideration of the alternatives could allow for tests of the long term effects of low diversity [e.g. mutational meltdown (Lynch et al., 1995)], independent of tests on the effects of a recent reduction in diversity (e.g. inbreeding depression).

In conclusion, our data suggest that, when possible, a historic sample of the endangered species or population in question should be used as the reference group. Even small archival collections may allow significance testing, although the variance of $\theta$ is inversely correlated with sample size (Tajima, 1983; Slatkin, 1987; Luikart et al., 1998). Importantly, studies of historical population structure may be useful in identifying appropriate reference populations. Historic samples can be drawn from any geographic locality that was not genetically subdivided from the ancestors of the population that eventually experienced a demographic bottleneck. Ultimately, if archival specimens are not available and no contemporary sample is suitable, then inferences regarding reductions in genetic diversity and increases in inbreeding should be appropriately tempered. A historical perspective, in combination with captive breeding data in this or other model systems (e.g. California Condor, Black-Footed Ferret), may allow for empirical examinations of the interaction between long term low genetic diversity, recent reductions in diversity, and fitness.

\section{Acknowledgements}

We thank George Roderick and Rosemary Gillespie while at the University of Hawaii; James L. Patton and the Museum of Vertebrate Zoology, U.C. Berkeley; and Jan A. Randall, San Francisco State University for providing space in their labs. Thanks also to Cristian Orrego for advise on laboratory techniques. We would also like to thank Andrew Bohanak, Susan Elrod, Roger Gambs, Eileen A. Lacey, James L. Patton, Aryan Roest, Javier Rodriguez, Kelly Zamudio, Diogo Meyer and Shannon McWeeney for comments on previous drafts of this manuscript. We thank the Museum of Vertebrate Zoology, California Polytechnic State University, and the National Zoological Park for tissues. 
Financial support was provided by NSF Awards \#9510822, USDA NRI Award \# 9601922, the American Wildlife Research Foundation and an American Museum of Natural History Theodore Roosevelt Grant.

\section{References}

Arctander, P., Johansen, C., Coutellec-Vreto, M.A., 1999. Phylogeography of three closely related bovid species (tribe Alcelaphini). Mol. Biol. Evol. 16, 1724-1739.

Avise, J.C., Shapira, J.F., Daniel, S.W., Aquadro, C.F., Lansman, R.A., 1983. Mitochondrial DNA differentiation during the speciation process in Peromysus. Mol. Biol. Evol. 1, 38-56.

Ballou, J.D., 1997. Ancestral inbreeding only minimally affects inbreeding depression in mammalian populations. J. Heredity 88 , 169-178.

Birky, C.W., Fuerst, P., Maruyama, T., 1989. Organelle gene diversity under migration, mutation, and drift: equilibrium expectations, approach to equilibrium, effects of heteroplasmic cells, and comparison to nuclear genes. Genetics 121, 613-628.

Bouzat, J.L., Lewin, H.A., Paige, K.N., 1998. The ghost of genetic diversity past: historical DNA analysis of the greater prairie chicken. Am. Nat. 152, 1-6.

Grassly, N.C., Adachi, J., Rambaut, A., 1997. PSeq-Gen: an application for the Monte Carlo simulation of protein sequence evaluation along phylogenetic trees. CABIOS 13, 559-560.

Hall, E.R., 1981. The Mammals of North America, 2nd Edition. Wiley, New York (pp. 573-579).

Hedrick, P.W., Miller, P.S., 1992. Conservation genetics: techniques and fundamentals. Ecol. Applic. 2, 30-46.

Hoelzel, A.R., Halley, J., O’Brien, S.J., Campagna, C., Arnbom, T., Le Boeuf, B. et al., 1993. Elephant seal genetic variation and the use of simulation models to investigate historical population bottlenecks. J. Heredity 84, 443-449.

Houlden, B.A., England, P.R., Taylor, A.C., Greville, W.D., Sherwin, W.B., 1996. Low genetic variability of the koala Phascolarctos cinereus in south-eastern Australia following a severe population bottleneck. Mol. Ecol. 5, 269-281.

Kuhner, M.K., Yamato, J., Felsenstein, J., 1998. Maximum likelihood estimation of population growth rates based on the coalescent. Genetics 149, 429-434.

Lacy, R.C., 1997. Importance of genetic variation to the viability of mammalian populations. J. Mamm. 78, 320-335.

Lande, R., 1988. Genetics and demography in biological conservation. Science 241, 1455-1460.

Lewontin, R.C., 1991. Twenty-five years ago in genetics: electrophoresis in the development of evolutionary genetics, milestone or millstone? Genetics 128, 657-662.

Li, W., Graur, D., 1991. Fundamentals of Molecular Evolution. Sinauer Associates, Sunderland, MA (p. 86).

Lindahl, T., 1993. Instability and decay of the primary structure of DNA. Nature 362, 709-715.

Luikart, G., Sherwin, W.B., Steele, B.M., Allendorf, F.W., 1998. Usefulness of molecular markers for detecting population bottlenecks via monitoring genetic change. Mol. Ecol. 7, 963-974.

Lynch, M., Conery, J., Burger, R., 1995. Mutation accumulation and the extinction of small populations. Am. Nat. 146, 489-518.

Maldonado, J.E., Davila, F.O., Stewart, B.S., Geffen, E., Wayne, R.K., 1995. Intraspecific genetic differentiation in California sea lions (Zalophus californianus) from southern California and the Gulf of California. Marine Mammal Sci. 11, 46-58.

McKnight, M.L., 1995. Mitochondrial DNA phylogeography of Perognathus amplus and Perognathus longimembris (Rodentia: Heteromyidae): a possible mammalian ring species. Evolution 49, 816-826.
Merola, M., 1994. A reassessment of homozygosity and the case for inbreeding depression in the cheetah, Acinonyx jubatus: implications for conservation. Conserv. Biol. 8, 961-971.

Merriam, C.H., 1907. Descriptions of ten new kangaroo rats. Proc. Biol. Soc. Washington 20, 75-79.

Moritz, C., 1994. Applications of mitochondrial DNA analysis in conservation: a critical review. Mol. Ecol. 3, 401-411.

Newman, D., Pilson, D., 1997. Increased probability of extinction due to decreased genetic effective population size: experimental populations of Clarkia pulchella. Evolution 51, 354-362.

Nusser, J.A., Goto, R.M., Ledig, D.B., Fleisher, R.C., Miller, M.M., 1996. RAPD analysis reveals low genetic variability in the endangered light-footed clapper rail. Mol. Ecol. 5, 463-472.

O’Brien, S.J., Wildt, D.E., Bush, M., Caro, T.M., Fitz-Gibbon, C., Aggundey, I. et al., 1987. East African cheetahs: Evidence for two population bottlenecks? Proc. Natl. Acad. Sci. USA 84, 508-511.

Patton, J.L., Smith, M.F., 1994. Paraphyly, polyphyly, and the nature of species boundaries in pocket gophers (genus Thomomys). Syst. Biol. 43, 11-26.

Patton, J.L., da Silva, M.N.F., Malcolm, J.R., 1994. Gene genealogy and differentiation among arboreal spiny rats (Rodentia: Echimyidae) of the Amazon basin: a test of the riverine barrier hypothesis. Evolution 48, 1314-1323.

Petit, E., Excoffier, L., Mayer, F., 1999. No evidence of bottleneck in the post glacial recolonization of Europe by the noctule bat ( $\mathrm{NyC}$ talus noctula). Evolution 53, 1247-1258.

Ralls, K., Ballou, J.D., Templeton, A.R., 1988. Estimates of lethal equivalents and the cost of inbreeding in mammals. Conserv. Biol. 2, 185-193.

Saccheri, I., Kuussaari, M., Kankare, M., Vikman, P., Fortelius, W., Hanski, I., 1998. Inbreeding and extinction in a butterfly metapopulation. Nature 392, 491-494.

Slatkin, M., 1987. The average number of sites separating DNA sequences drawn from a subdivided population. Theor. Pop. Biol. $32,42-49$

Steinberg, E.K., Jordan, C., 1998. Using molecular genetics to learn about the ecology of threatened species: The allure and the illusion of measuring genetic structure in natural populations. In: Fiedler, P.L., Kareiva, P.M. (Eds.), Conservation Biology, 2nd Edition. Chapman and Hall, New York, pp. 440-460.

Stewart, G.R., Roest, A.I., 1960. Distribution and habits of kangaroo rats at Morro Bay. J. Mamm. 41, 126-129.

Taberlet, P., Fumagalli, L., Hausser, J., 1994. Chromosomal versus mitochondrial DNA evolution: tracking the evolutionary history of the southwestern European populations of the Sorex araneus group (Mammalia, Insectivora). Evolution 48, 623-636.

Tajima, F., 1983. Evolutionary relationship of DNA sequences in finite populations. Genetics 105, 437-460.

Tajima, F., 1989. Statistical method for testing the neutral mutation hypothesis by DNA polymorphism. Genetics $123,585-595$.

Taylor, A.C., Sherwin, W.B., Wayne, R.K., 1994. Genetic variation of microsatellite loci in a bottlenecked species: the northern hairynosed wombat Lasiorhinus krefftii. Mol. Ecol. 3, 277-290.

Thomas, W.K., Pääbo, S., Villablanca, F.X., Wilson, A.C., 1990. Spatial and temporal continuity of kangaroo rat populations shown by sequencing mitochondrial DNA from museum specimens. J. Mol. Evol. 31, 101-112.

US Fish and Wildlife Service, 1995. Morro Bay Kangaroo Rat Draft Revised Recovery Plan. Portland, Oregon.

Villablanca, F.X., 1994. Evolutionary analysis: spatial and temporal aspects of populations revealed by mitochondrial DNA. In: Herrmann, B., Hummel, S. (Eds.), Ancient DNA: Recovery and Analysis of Genetic Material from Paleontological, Archaeological, Museum, Medical, and Forensic Specimens. Springer-Verlag, New York, pp. 31-58.

Watterson, G., 1975. On the number of segregating sites in genetical models without recombination. Theor. Popul. Biol. 7, 256-276. 
Wayne, R.K., Jenks, S.M., 1991. Mitochondrial DNA analysis implying extensive hybridization of the endangered red wolf Canis rufus. Nature 351, 565-568.

Wilmer, J.W., Hall, L., Barratt, E., Moritz, C., 1999. Genetic structure and male-mediated gene flow in the ghost bat (Macroderma gigas). Evolution 53, 1582-1591.

Wright, S., 1969. Evolution and the Genetics of Populations. University of Chicago Press, Chicago. 